\title{
Research Paper \\ Prevalence and Factors Related to Self-Medication for COVID-19 Prevention in the Elderly
}

\author{
Narjes Heshmatifar ${ }^{1}\left(\mathbb{C}\right.$, Arezoo Davarinia Motlagh Quchan², Zohreh Mohammadzadeh Tabrizi ${ }^{2}$, Leila Moayed ${ }^{3}\left(\mathbb{C}\right.$, Sajad $^{2}$ \\ Moradi ${ }^{4}$, Sedighe Rastagi ${ }^{5}{ }^{\circ}$, ${ }^{*}$ Fatemeh Borzooei ${ }^{6}$ ㄴ)
}

1. Department of Nursing, Aging Research Center, School of Nursing and Midwifery, Sabzevar University of Medical Sciences, Iran.

2. Department of Anesthesiology, Student Research Committee, School of Paramedics, Sabzevar University of Medical Sciences, Iran.

3. Department of Geriatric Nursing, School of Nursing, Sabzevar University of Medical Sciences, Iran.

4. Vasei Hospital, Sabzevar University of Medical Sciences, Sabzevar, Iran.

5. Department of Biostatistics, Student Research Committee, School of Health, Mashhad University of Medical Sciences, Mashhad, Iran.

6. Department of Operating Room, Aging Research Center, School of Paramedics, Sabzevar University of Medical Sciences, Iran.

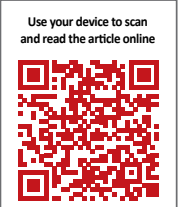

Citation: Heshmatifar N, Davarinia Motlagh Quchan A, Mohammadzadeh Tabrizi Z, Moayed L, Moradi S, Rastagi S, et al. [Prevalence and Factors Related to Self-Medication for COVID-19 Prevention in the Elderly (Persian)]. Iranian Journal of Ageing. 2021; 16(1):112-127. https://doi.org/10.32598/sija.16.1.2983.1

doi https://doi.org/10.32598/sija.16.1.2983.1

Key words:

Drug, Elderly, Preva-

lence, COVID-19,

Self-medication

\section{A B STRACT}

Objectives The Coronavirus Disease 2019 (COVID-19) crisis and health outcomes are among the major human social events of the 21st century. The unknown nature of the disease and the fear of contagion have caused emotional reactions, including the self-consumption of drugs in the elderly. Therefore, this study investigated factors affecting the self-consumption of drugs for COVID-19 prevention in the elderly. Methods \& Materials This descriptive and cross-sectional study was performed on 342 elderly in Sabzevar City, Iran, in 2020. The research units were selected as clusters from 16 health centers. The necessary data were collected by an online self-medication questionnaire. Data analysis was performed in SPSS.

Results The Mean $\pm S D$ age of the study participants was $66.2 \pm 5.67$ years. The frequency of self-medication to prevent COVID-19 was 190(55.5\%); analgesics, vitamins, anti-cold, and antibiotics were the most common drugs used. The major factors associated with self-medication in the COVID-19 pandemic were disease prevention, home quarantine, financial problems, experiencing previous self-medication, and others' advice. There was a significant relationship between self-medication and education and insurance coverage. No significant relationship was observed between gender, occupation, and marital status, and self-medication.

Conclusion Self-Medication in the COVID-19 epidemic is notable as a crisis threatening the elderly's health. Controlling and monitoring how the drug is used is among the requirements for protection and health promotion in the elderly.

\section{Extended Abstract}

\section{Introduction}

I n 2020, the World Health Organization declared the outbreak of Coronavirus Disease 2019 (COVID-19), as a global emergency [1]. All age groups are at risk for coronavirus infection; however, the elderly are more vulnerable for various reasons, including deficits in immune systems, chronic diseases, poor personal hygiene, loneliness, multi drugs use, and the lack of medical attention [2]. Morbidity, mortality, the lack of specific treatment for COVID-19 [3], and psychological problems, like death anxiety, have led the elderly to arbi-

*Corresponding Author:

Fatemeh Borzooei

Address: Department of Paramedics \& Department of Operating Room, Aging Research Center, Faculty Member, Sabzevar University of Medical Sciences, Iran.

Tel: +98 (51) 44018325

E-mail: borzoee75026@yahoo.com 
trarily use drugs [4]. Studies suggested that the elderly lack the necessary knowledge about the adverse effects of selfmedication; thus, they must acquire sufficient knowledge to change behavior [5]. Despite the importance of pharmacoepidemiological studies, such studies were not found in critical conditions. Due to the aging trend of the population of the country, the present study aimed to investigate factors related to the arbitrary use of drugs for COVID-19 prevention in the elderly.

\section{Methods and Materials}

This descriptive and cross-sectional study was performed on 360 elderly (aged $>60$ years). Based on self-reported data, the selected elderly had no acute physical or cognitive problems.

The samples were identified by a random cluster sampling method. They were determined from the population areas of 16 comprehensive health centers in Sabzevar City, Iran; the city was divided into 4 regions. Two centers were randomly selected per region. Then, a sample size appropriate to the number of the elderly per center was selected from each center. Given the special conditions of COVID-19 and the impossibility of face-to-face communication, the researcher used the WhatsApp platform to form a group among the elderly; accordingly, the required data were collected remotely. In addition to completing the online questionnaire, the research units were requested to mention the names of the drugs used or to send a picture of the drug package. The questionnaire included a section where informed the elderly that they could leave the study freely.

To collect the necessary data, a demographic form and a researcher-made questionnaire were used. These tools examined the self-medication status of the elderly during the last 3 months in the COVID-19 crisis. The explored elderly's self-medication status consisted of 21 items, including the history and type of self-medication during the COVID-19 pandemic (4 items), awareness of the type of medication (3 items), self-medication situations ( 7 items), and medications used to prevent COVID-19 (7 items). The items were answered on a 5-point Likert-type scale. The validity of the questionnaire was confirmed by the content validity method and its face validity was also approved as desirable. The reliability of the questionnaire was determined by the internal consistency reliability method and its Cronbach's alpha coefficient was measured as $89 \%$. Descriptive statistics and Chi-squared test were used to evaluate self-medication and qualitative variables. The obtained data were analyzed using SPSS at a significance level of 0.05 . This research was approved by the Ethics Committee of Sabzevar University of Medical Sciences (Code: IR.MEDSAB.REC.1399.020).

\section{Results}

The study sample included 152(44.5\%) males and $190(55.5 \%)$ females; the Mean \pm SD age of the study participants was $66.2 \pm 5.67$ years. Moreover, $57.3 \%$ of the study samples were married and $42.7 \%$ were single. Furthermore, $29.8 \%$ of the study participants were illiterate, $54.5 \%$ had an elementary education, and $15.7 \%$ had a high school diploma or higher. Besides, $31.3 \%$ of the study samples were retired, $21.6 \%$ were self-employed, and $47.3 \%$ were unemployed. Additionally, $30.4 \%$ of the study subjects used no health insurance, $52.7 \%$ used regular insurance, and 16.9\% used supplementary insurance.

The Chi-square test data indicated a significant relationship between self-medication, and education and insurance coverage $(\mathrm{P}<0.05)$. However, this test presented a signifi-

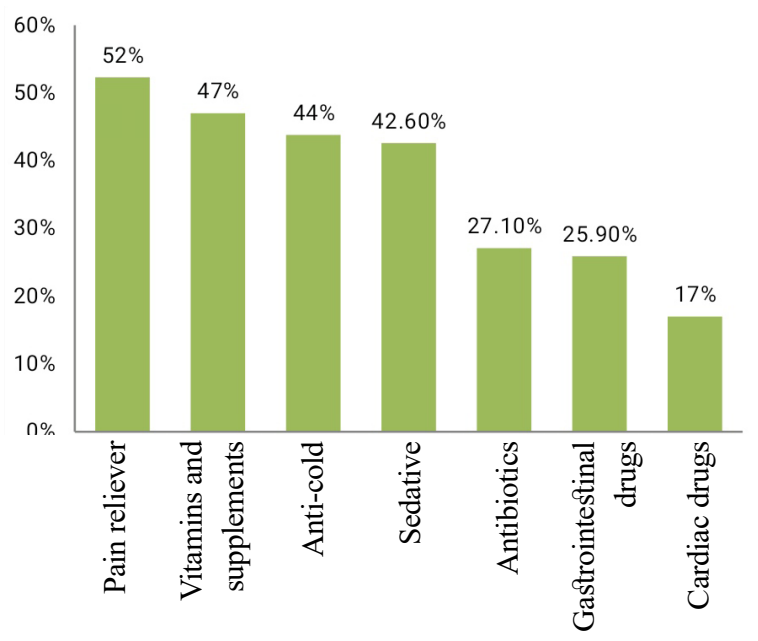

Figure 1. The frequency of drugs used for self-medication among the explored elderly in the COVID-19 pandemic 
Table 1. Reasons for self-medication during COVID-19 pandemic in the studied elderly

\begin{tabular}{|c|c|}
\hline Variable & No. (\%) \\
\hline Prevention of coronavirus & $101(52.3)$ \\
\hline Avoid going out (home quarantine) & $99(51.5)$ \\
\hline Unable to afford a visit or pay for medication & $53(27.4)$ \\
\hline Previous experience of taking the drug & $49(25.3)$ \\
\hline Recommended by others, pharmacy, media & $45(23.3)$ \\
\hline The lack of insurance coverage & $33(17)$ \\
\hline Mild symptoms & $32(16.5)$ \\
\hline Easy access to medicines (available at home, cheap drugs, over-the-counter sales in pharmacies) & $30(15.5)$ \\
\hline Disbelief in common treatment methods & $21(10.8)$ \\
\hline Staying away from physicians' offices & $14(7.2)$ \\
\hline The lack of knowledge about the arbitrary adverse effects of the drug & $12(6.2)$ \\
\hline Not having enough time to visit a physician & $10(5.1)$ \\
\hline
\end{tabular}

cant relationship between gender, occupation, and marital status, and self-medication $(\mathrm{P}>0.05)$.

The collected results revealed that the rate of arbitrary drug use during the COVID-19 pandemic in the elderly equaled 193(56.4\%). Despite self-medication in more than $50 \%$ of units of this study, only $13.3 \%$ of them had the minimum necessary drug information (drug name, classification, uses, \& adverse effects). Moreover, 53.8\% of the research units provided drugs from home and $46.2 \%$ from pharmacies. Furthermore, 98(50.7\%) of the explored elderly used drugs in the form of tablets or capsules, $25.3 \%$ used syrups, $11.9 \%$ used ampoules, and $11.9 \%$ used other forms of drugs. The type of knowledge of most of them about self-medication based on the category of drugs was $39.1 \%$; the same rate for sending a package photo of the drugs was $37.7 \%$, and $23.2 \%$ of the samples mentioned the name of the drug used.

In evaluating the conditions and situations of self-medication in the examined elderly, $43.4 \%$ related to joint and muscle pain, $42 \%$ neurological diseases, $41.9 \%$ pseudocorona symptoms, $31 \%$ weakness and lethargy, and $21 \%$ headache. The prevalence of gastrointestinal conditions and cardiovascular disorders were $20 \%$ and $12 \%$ in the study subjects, respectively. The highest drug classes in arbitrary use in the research units were analgesics, tonics, anti-colds, and sedatives, orderly (Figure 1).

The main reasons for the arbitrary use of the drug during the COVOD-19 pandemic in the elderly who reported self-medication respectively concerned COVID-19 prevention, as the most common cause $(52 \%)$, followed by selfmedication for other reasons, such as home quarantine and avoiding going out, not affording a physician visit, previous experience in the use of drugs, and others' advice (Table 1).

\section{Discussion and Conclusion}

The present study results suggested that approximately 1.2 of the explored elderly practiced self-medication during the COVID-19 pandemic; thus, they felt at risk of contracting the coronavirus. Reducing the fear of the elderly by raising awareness and providing accurate information about $\mathrm{CO}$ VID-19 is essential by creating a campaign in public and virtual media. Self-medication has also been reported in the educated and uninsured elderly. It is recommended that those in charge of drug supply take all considerations, including refraining from dispensing over-the-counter drugs and providing training on the arbitrary use of drugs in old age when purchasing drugs. Due to the possible problems and risks due to arbitrary drug use in the COVID-19 pandemic, it seems necessary to provide special training programs for the elderly in healthcare centers. Therefore, it is recommended that the authorities take action to reduce this problem to raise the elderly's awareness. 


\section{Ethical Considerations}

\section{Compliance with ethical guidelines}

This research was approved by the Ethics Committee of Sabzevar University of Medical Sciences (Code: IR.MEDSAB.REC.1399.020). All ethical principles are considered in this article. The participants were informed about the purpose of the research and its implementation stages. They were also assured about the confidentiality of their information and were free to leave the study whenever they wished, and if desired, the research results would be available to them.

\section{Funding}

This study was extracted from a research project of Nursing School from Sabzevar University of Medical Sciences.

\section{Authors' contributions}

Conceptualization, supervision, writing - review \& editing: Narjes Heshmatifar, Fatemeh Borzoee; Methodology, review: Arezoo Davarinia Motlagh Quchan, Zohreh Mohammadzadeh Tabrizi, Sajad Moradi; Data collection: Leila Moayed; Data analysis: Sedighe Rastaghi.

\section{Conflicts of interest}

All authors declare no conflict of interest.

\section{Acknowledgements}

We would like to thank all staff and officials in Sabzevar School of Nursing and health centers in Sabzevar are thanked. 
This Page Intentionally Left Blank 


\title{
شيوع و عوامل مرثبط با خوددرمانى در ييشَّيرى از كوويد 9 أدر سالمندان
}

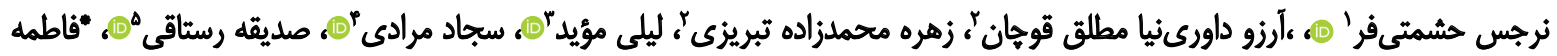

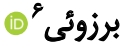

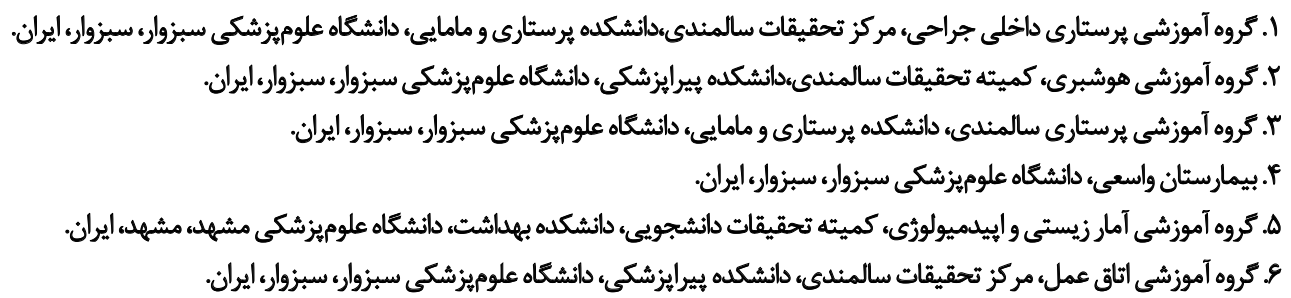

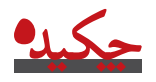

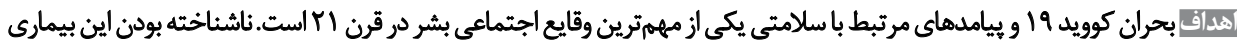

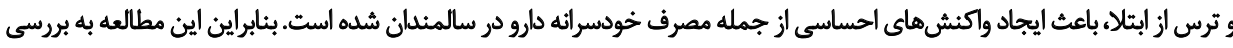

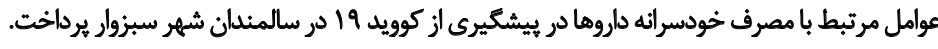

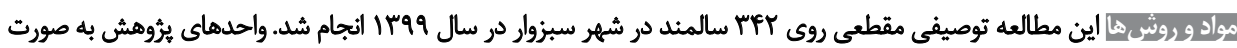

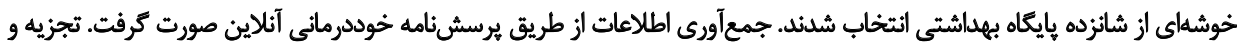

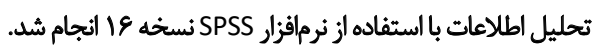

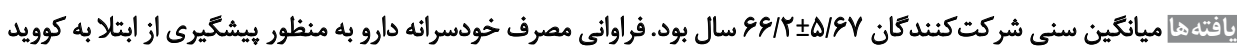

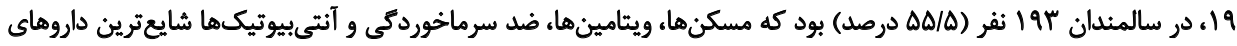

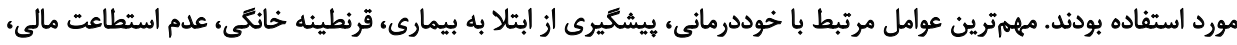

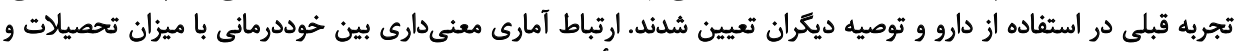

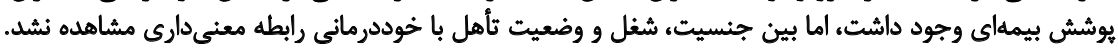

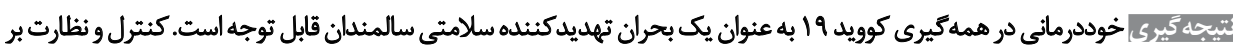

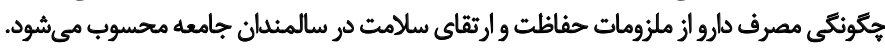

تاريخ دريافت: 14 اخرداد

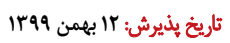

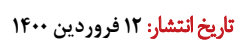

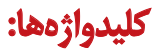

داروه سالمندان، شيوع، كوويد 19 1ا، خوددرمائي

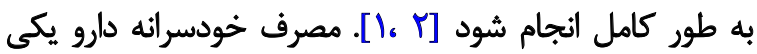

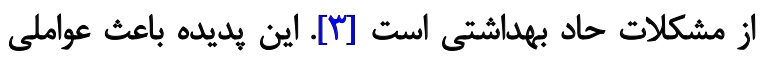

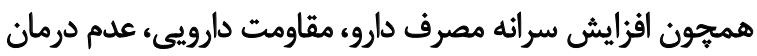

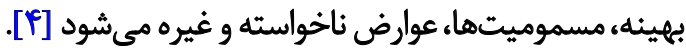

امروزه دسترسى آسان به داروهاى بدون نسخه يا با نسخه،

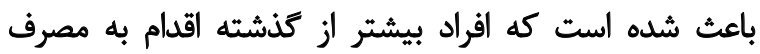

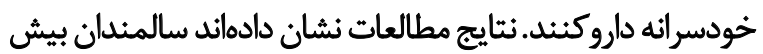

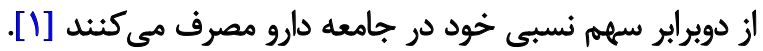

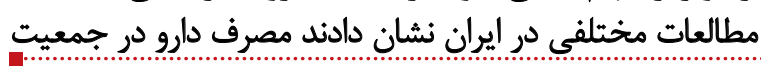

doles

خوددرمانى به عنوان معمولى ترين فرم خودمراقبتى مي دتواند از

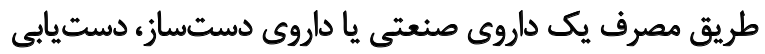

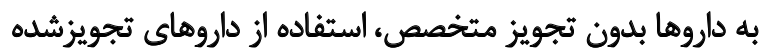

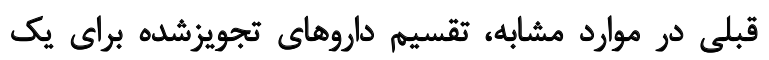

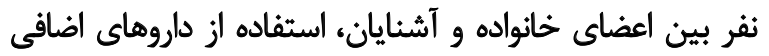

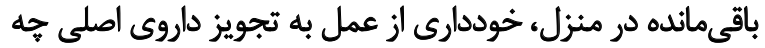

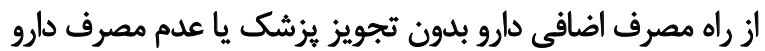

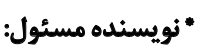

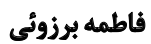

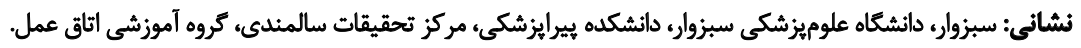

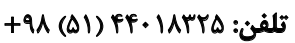
يست الكترونيكي: borzoee75026@yahoo.com 
موربيديتى و مورتاليتى و سرعت واكيرى بالاي كروناويروس و

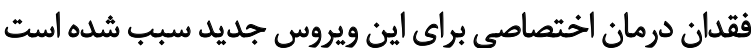

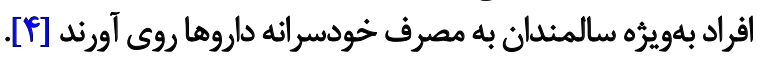

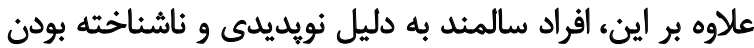

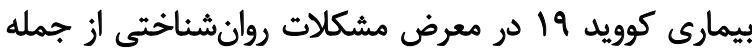
استرس، افسردكى و اضطراب مركى قرار كرفتهاند و واين امر نيز

منجر به خوددرمانى و افزايش داروهاى مصرفى مى مشود [ [ I ].

مصرف داروهاى متعدد در سالمندان عوارضى به دنبال دارد

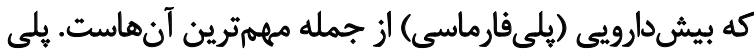

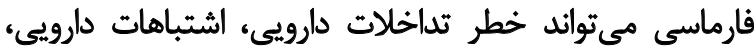

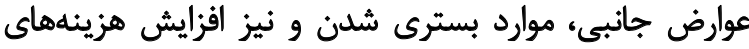

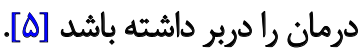

بادر نظر كرفتن اين موضوع كه سالمندى مي تواند به طور خاص

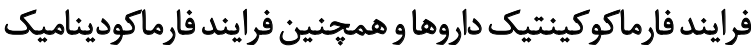

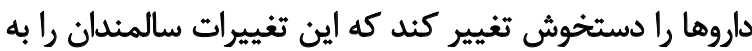

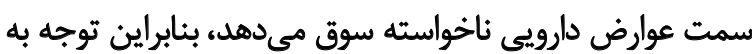
اين قشر از جامعه در شرايط خاص و بحرانى ضرورى بـ به نظر بنائر

بررسى هانشان دادهاند اكثر افراد جامعه و به خصوص سالمندان

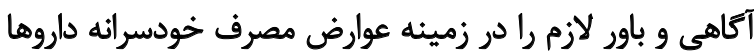

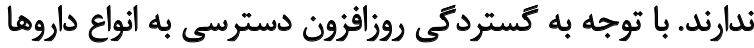

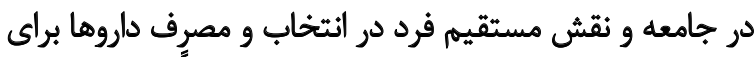

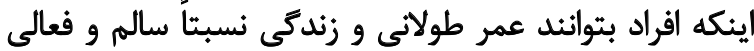

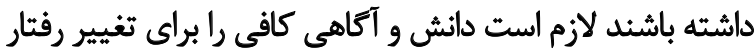

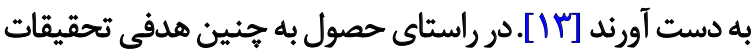

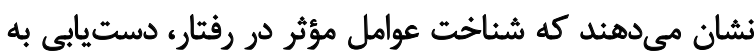

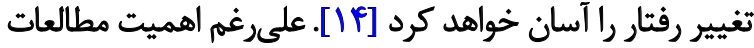

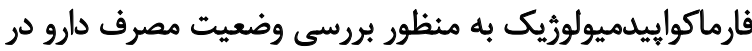

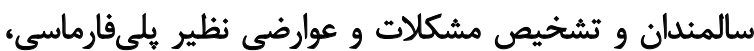

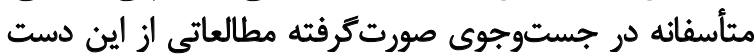

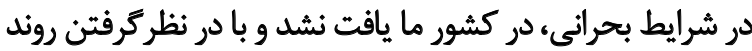

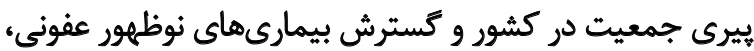

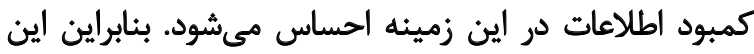

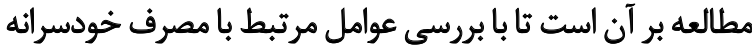

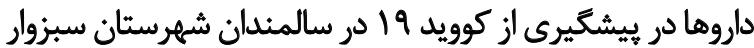

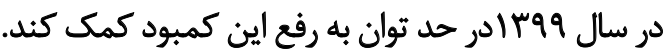

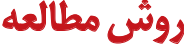

اين مطالعه توصيفى مقطعى روى •وب سالمند (بالاى إي

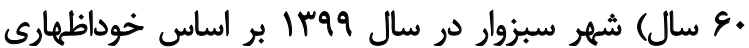

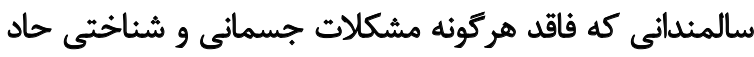

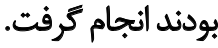

امروزى و بلويره در افراد مسن در طي دهلهاي اخير افزايش

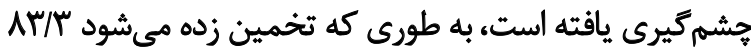

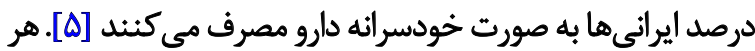

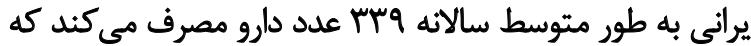

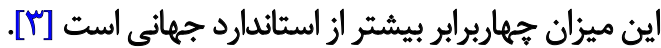
دريافت داروى نامناسب براي سالمندان يك مسئله مههم است،

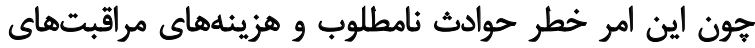

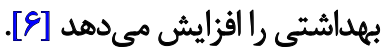

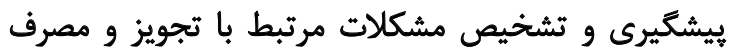

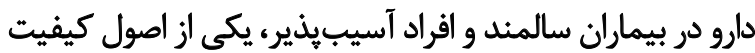

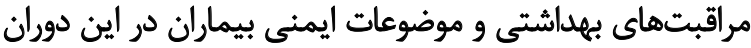

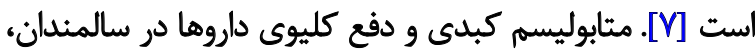

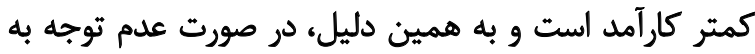

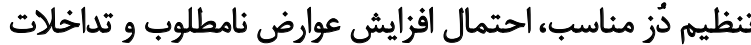
دارويى افزايش مىيابد [1]]. در شرايط بحران و شيوع بيمارىها، به دليل استرس، اضطراب،

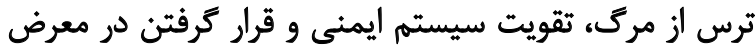

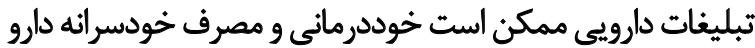

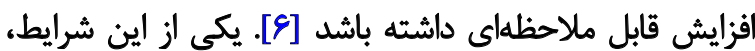
بيمارى نوظهوركروناويروس يا كوويد 919 است.

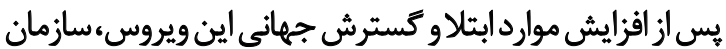

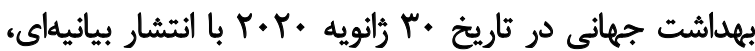

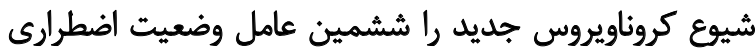

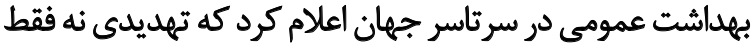

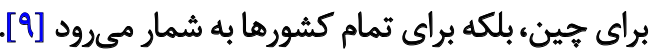

اين عفونت ويروسى باعث بروز بيمارى حاد تنفسى با علاثمي

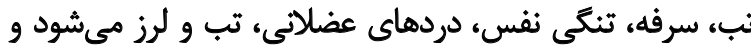

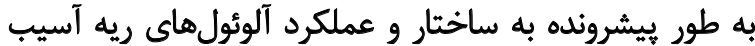

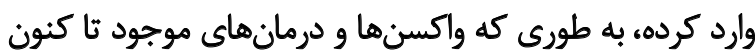

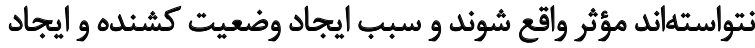

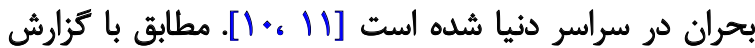

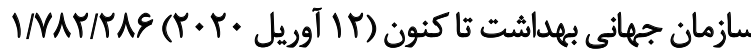

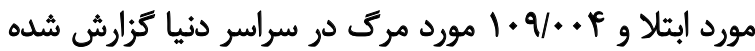

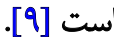

هرجند تمام كروههاى سنى در معرض ابتلا به كروناويروس

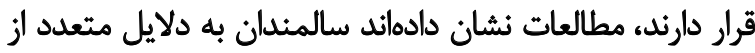

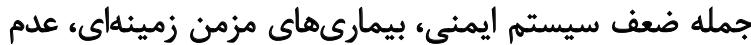

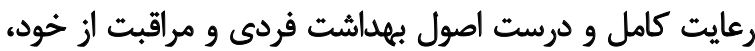

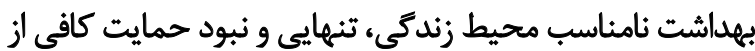

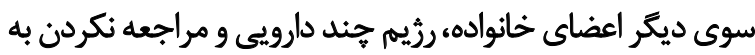

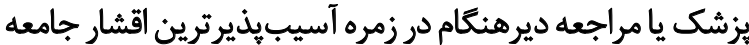

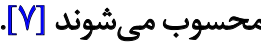


حضورى، يروهشكَر با استفاده از فضاى مجازى تلفن همراه

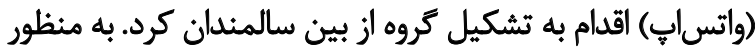

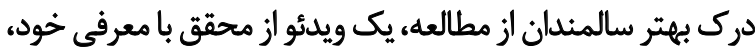

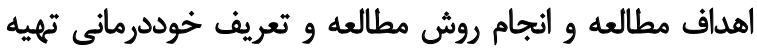

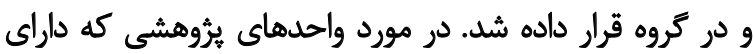

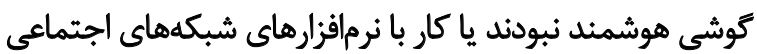

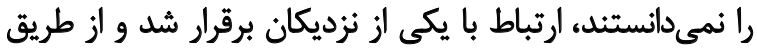

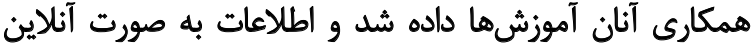

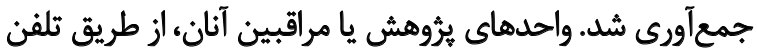

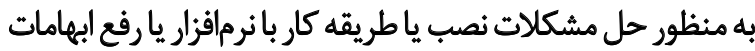

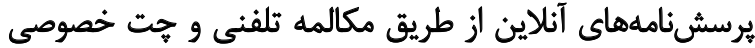

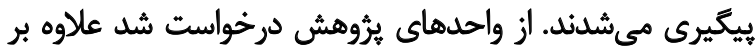

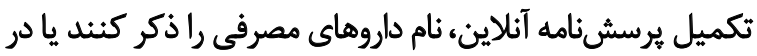

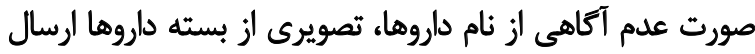

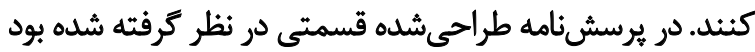

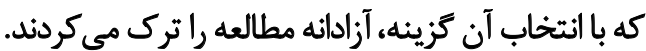

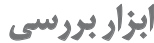

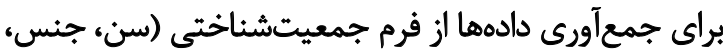

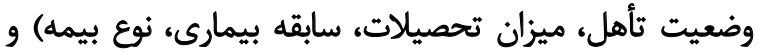

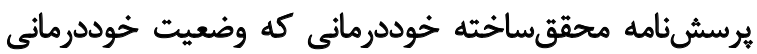

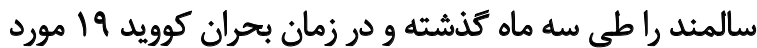

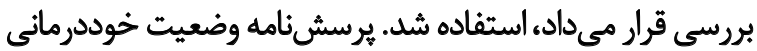

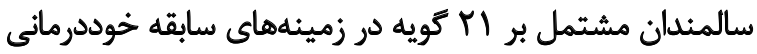

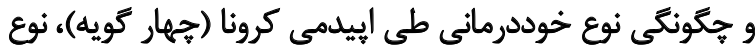

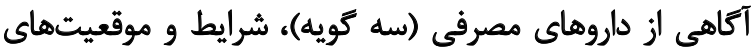

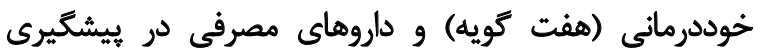

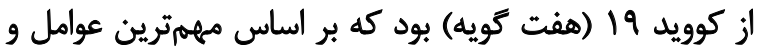

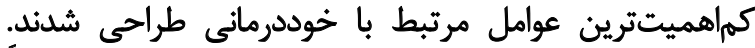

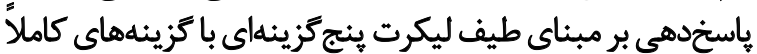

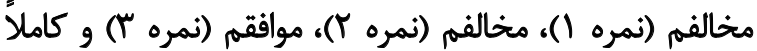

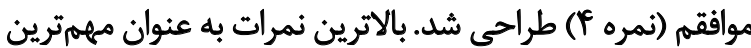

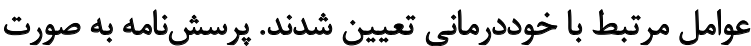

$$
\text { آنلاين طراحى شرتيط باخدودر. }
$$

سؤالات در جندين مرحله بازبينى شدند و جهت تأييد اعتبار

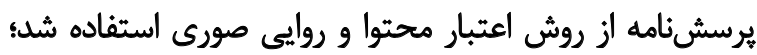

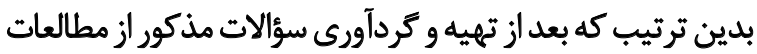

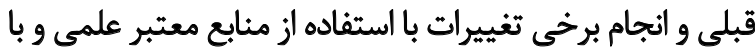

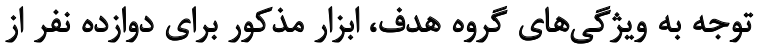

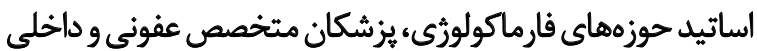

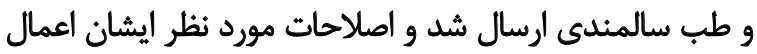

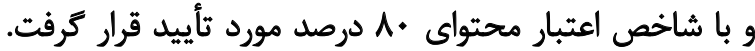

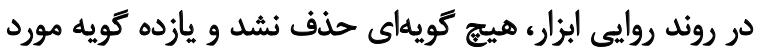

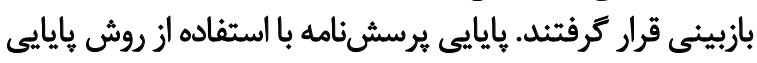

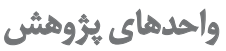

جامعه مورد يرؤوشش شامل سالمندان تحت يوشش مراكز

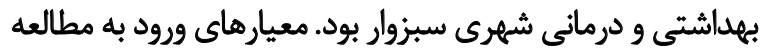

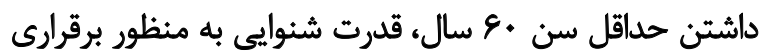

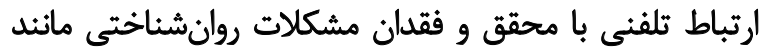

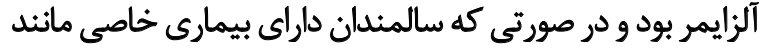

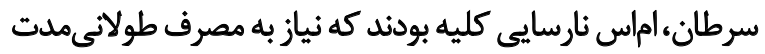

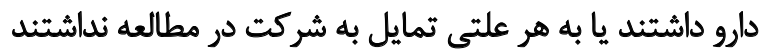

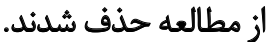

حجم نمونه

براى به دست آوردن حجم نمونه از فرمول شماره ا استفاده شد.

$$
n=\frac{N\left(z \frac{p}{2} \cdot p(1-p)\right.}{\varepsilon^{2}(N-1)+\frac{\left(z \frac{z}{2} \cdot p(1-p)\right.}{2}}
$$

$\mathrm{n} \equiv \frac{2301(1 / 96)^{2} \cdot 0 / 5(1-0 / 5)}{(0 / 5)^{2}(227-1)+(1 / 96)^{2} \cdot 0 / 5(1-}=330 / 126 \cong 330$

$(0 / 5)^{2}(227-1)+(1 / 96)^{2} \cdot 0 / 5(1-$

0/5)

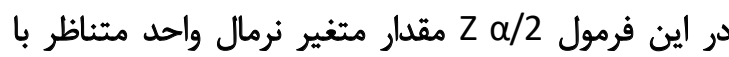

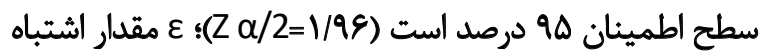
مجاز است كه مقدار آن بر اساس احتمال خطاي المان نمونه محاسبه

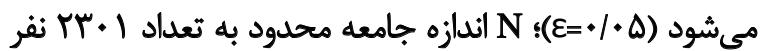

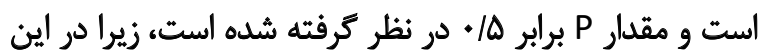

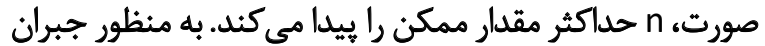

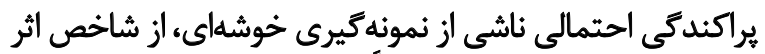

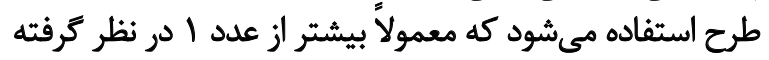

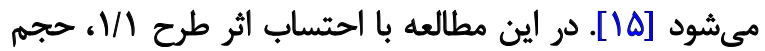
نمونه معادل ب بوس نهايي شد.

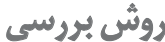

يّ إز اخذ مجوز از دانشعاه علوميزشكى سبزوار و ارائه

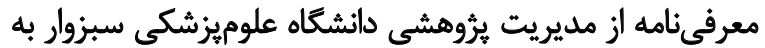

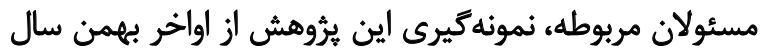

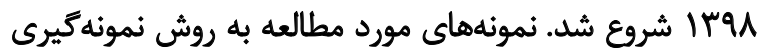

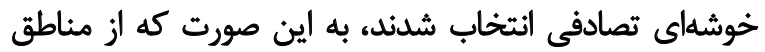

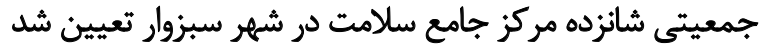

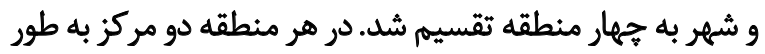

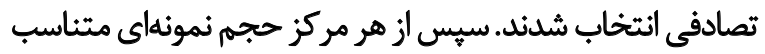

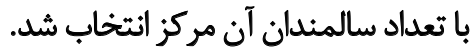
به دليل شرايط ويره بيمارى كرونا و عدم برقرارى ارتباط 
نتايج نشان داد ميزان مصرف خودسرانه دارو در دوران

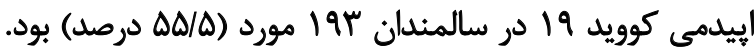

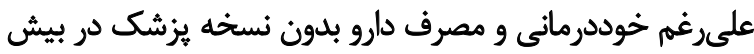

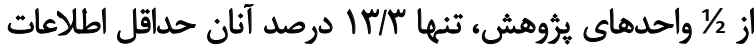

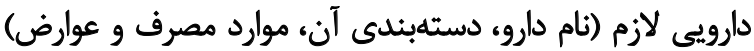

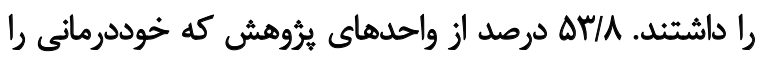

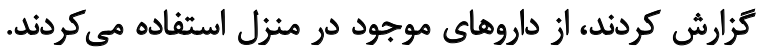

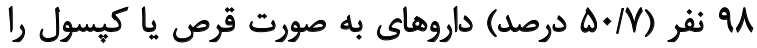

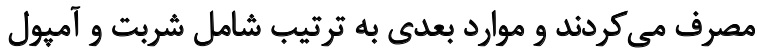

$$
\text { بود (جدول شماره r r). }
$$

همان أنور كه نتايج جدول شماره ب نشان مى دهد، نوع آكاهى

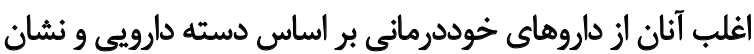

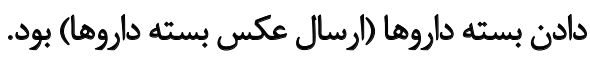

شرايط و موقعيتهاى خوددرمانى در سالمندان تحت مطالعه،

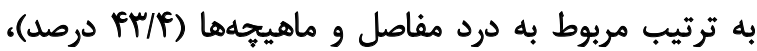

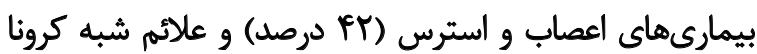

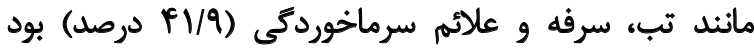

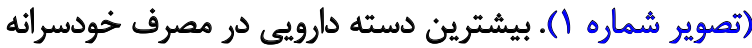

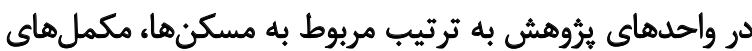

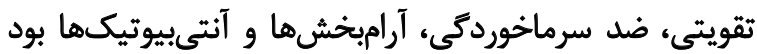

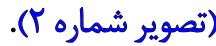
در ادامه بررسى ها، مهمترين دلايل مصرف خودسرانه دارو در
همسانى درونى بعد از تكميل برسشنامه توسط بيست نفر از

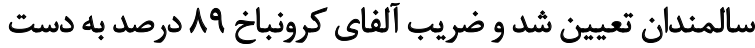

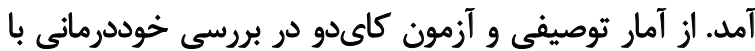

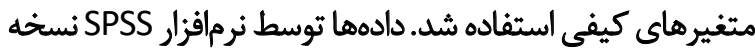

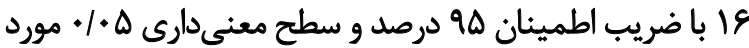
ت ت تجزيه و تحليل قرار كرفتند.

ياقتهها

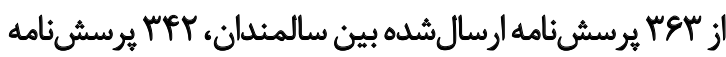

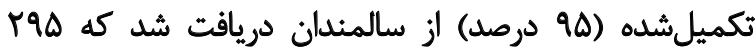

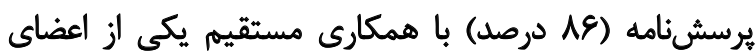

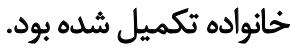

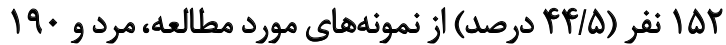

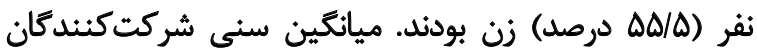

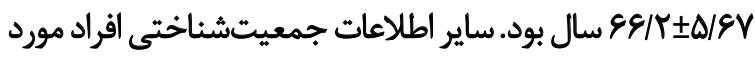

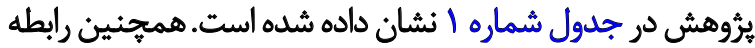

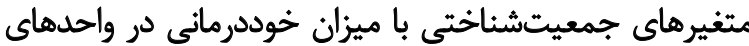

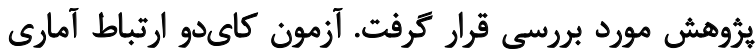

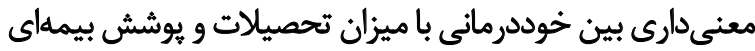

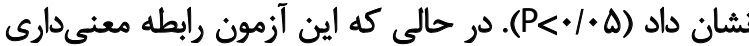

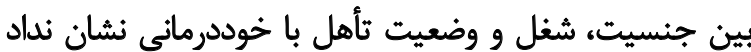

(P> $(1 \cdot \Delta)$

جدول ا. مشخصات جمعيتشناختى سالمندان سبزوار و ارتباط آنان با خوددرمانى

\begin{tabular}{|c|c|c|c|}
\hline سطح معنى دارى" & فراوانى (درصد) & متغير & \\
\hline \multirow{2}{*}{.$/ 191$} & $\operatorname{lar}(\varphi \varphi / q)$ & مرد & \multirow{2}{*}{ جنسيت } \\
\hline & $19 \cdot(\Delta \Delta / \Delta)$ & 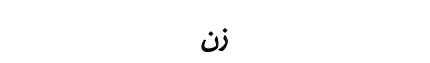 & \\
\hline \multirow{2}{*}{$\cdot / K \cdot 1$} & $1 q(\Delta V / r)$ & مثتأهل & \multirow{2}{*}{ وضعيت تأهل } \\
\hline & $\operatorname{lES}(T T M)$ & مجرد (بيوه يا مثاركه) & \\
\hline \multirow{3}{*}{$<+1++1$} & $1 . r(r / /)$ & ل بىسواد & \multirow{3}{*}{ تحصيلات } \\
\hline & $W \in(\Delta F / \Delta)$ & ابتلايى & \\
\hline & $\Delta f(1 \Delta / N)$ & دييلم و بالاتر & \\
\hline \multirow{3}{*}{$\cdot / \lambda \cdot 1$} & $1 \cdot \gamma(r / / r)$ & بازنشسته & \multirow{3}{*}{ شغل } \\
\hline & $n(r / g)$ & آزاد & \\
\hline & $\operatorname{IQT}(P V / T)$ & بيكار & \\
\hline \multirow{3}{*}{$<+1 \cdot+1$} & $1 . \varphi(\% \cdot / \varphi)$ & بدون يوشش بيمه درمانى & \multirow{3}{*}{ بيمه } \\
\hline & $M \cdot(\Delta Y / M)$ & 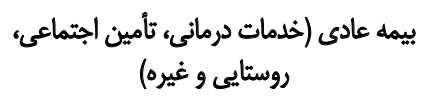 & \\
\hline & $\Delta \Lambda(19 / 9)$ & بيمه تكميلى & \\
\hline
\end{tabular}


جدول r. جَكونكى نوع خوددرمانى در سالمثدان شهر سبزوار در ابيدمى كرونا

\begin{tabular}{|c|c|c|}
\hline فراوانى (درصد) & & \\
\hline $19 \Psi(\Delta S / F)$ & بله & \multirow[b]{2}{*}{ خودرمانى } \\
\hline $1 F q(F r / \Delta)$ & خير & \\
\hline $\operatorname{IQV}(A \& /)$ & دارد & \multirow{2}{*}{ اطلاعات دارويى } \\
\hline$r \varepsilon(1 T / T)$ & ندارد & \\
\hline $1 . \psi(\Delta r / \Lambda)$ & مثنزل & \multirow{2}{*}{ نحوه تهيه دارو } \\
\hline $19(\% q / T)$ & داروخاثه & \\
\hline$u(\Delta \cdot / N)$ & قرص يا كيسول & \multirow{4}{*}{ شكل رايج دارو } \\
\hline$P q(r \Delta / r)$ & شريت & \\
\hline$\pi(11 / 9)$ & آميول & \\
\hline$r(11 / 9)$ & ساير اشكال دارويى & \\
\hline
\end{tabular}

$\stackrel{1}{2}$

جدول با. نوع آكاهى از ماروى مصرفى در سالمندان سبزوار در إيدمى كرونا

\begin{tabular}{|c|c|}
\hline فراواتى (درصد) & آكاهى از داروى مصرفى \\
\hline$(M / T)$ PA & ذ ذكر نام دارو \\
\hline$(r a / 1) V \Delta$ & ذكر دستلهبندى دارويى \\
\hline$(r V / N) n$ & نشان دادن بسته دارو \\
\hline
\end{tabular}

L

جدول F. دلايل خوددرمانى در إيدمى كوويد 19 در سالمندان شهر سبزوار

\begin{tabular}{|c|c|}
\hline (درصد) فراوانى & متغير \\
\hline $1.1(\Delta / / T)$ & ييشكيرى از ابتلا به كروناويروس \\
\hline $99(\Delta) / \Delta)$ & اجتناب از بيرون رفتن (قرنطينه خانكى) \\
\hline$\Delta r(T V / \varphi)$ & عدم استطاعت مالى براى يرداخت ويزيت يا هزينه داروها \\
\hline$F q(T \Delta / r)$ & تجربه قبلى مصرف دارو \\
\hline$F \Delta(\pi / \%)$ & توصيه ديكران، داروخانه، رسانهها \\
\hline$\pi(I V / \bullet)$ & علم يوشش بيمهاى \\
\hline$\pi r(1 \& / \Delta)$ & خفيف بودن علاثم بيمارى \\
\hline$r+(1 \Delta / \Delta)$ & 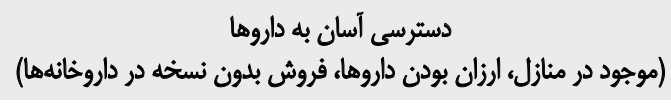 \\
\hline$M(1 \cdot / A)$ & عدم اعثقاد به درمانهاى رايج \\
\hline If $(V / T)$ & دورى از مطب يزشكان \\
\hline $\mathbb{I r}(\xi / r)$ & عدم آكاهى در مورد عوارض خودسرانه دارو \\
\hline $1 \cdot(\Delta / 1)$ & نداشتن وقت كافى جهت مراجعه به يزشك \\
\hline
\end{tabular}

$\stackrel{2}{2}$ 


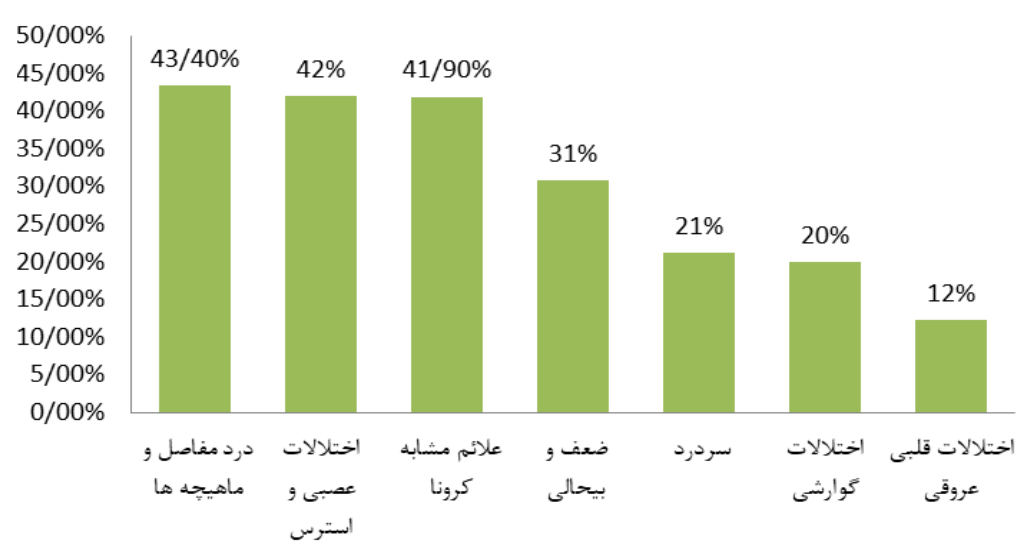

اقتصادى اجتماعى بين جوامع است. شيوع ويروس كرونا و

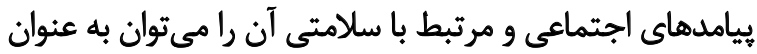

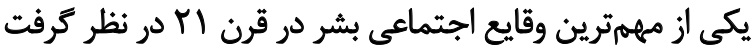

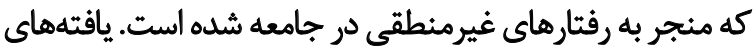

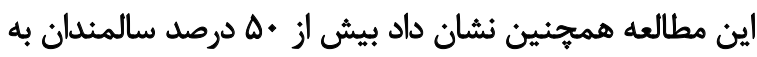
دليل ييشكيرى از ابتلا به بيمارى كوريد

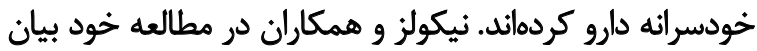

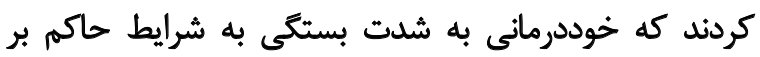

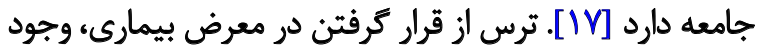

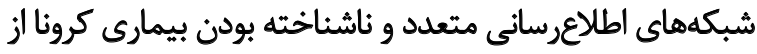

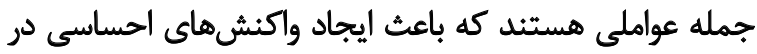

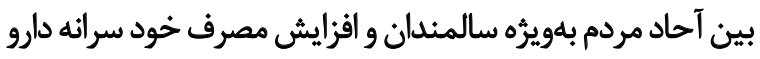

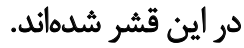

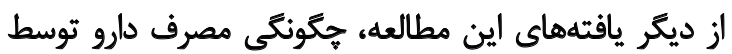

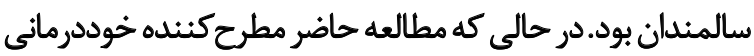
مشهود سالمندان اين يُروهش است، اما فقط تعداد كمى آز آنان

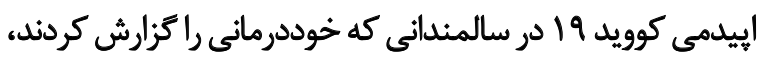

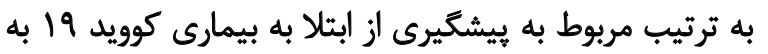

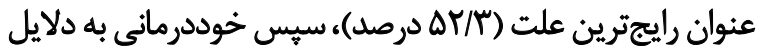

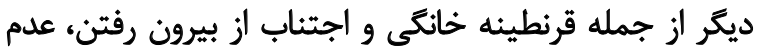

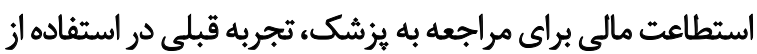
دارو و توصيه ديكران بود (جدول شماره \&). بحث

مطالعه حاضر با هدف بررسى شيوع و عوامل مرتبط بان

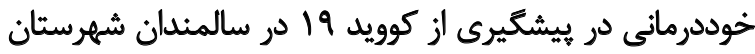

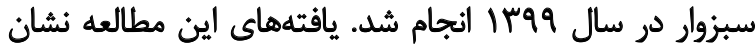

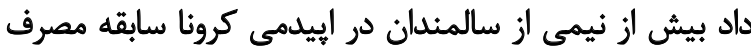

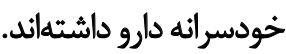
مطالعهاى كه توسط ماسوموتو و همكاران در راين انجام ترفته

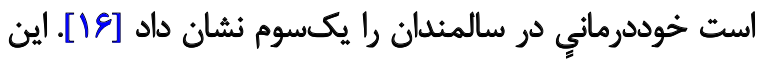

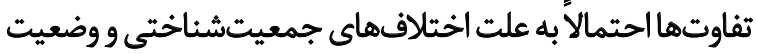

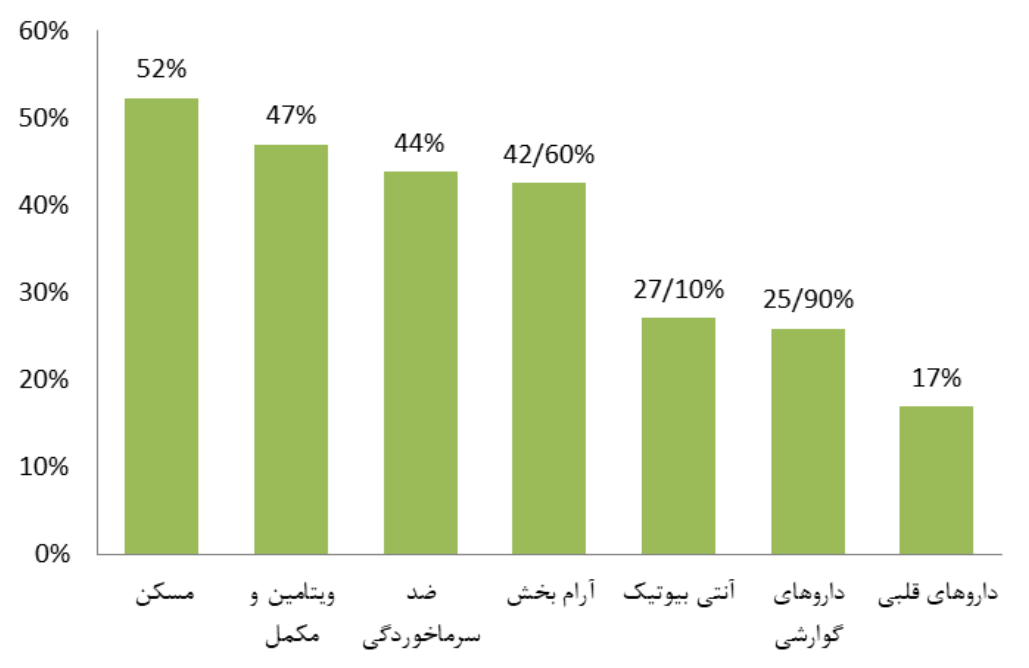


نتايج همجينين نشان داد به ترتيب، ترس از ابتلا به بيمارى،

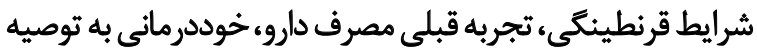

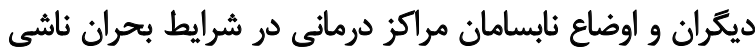

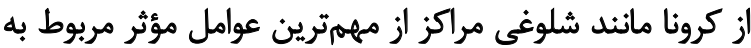

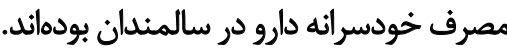

ماكوسكاو همكاران در مطالعه خود رفتارهاي مرتبط با مصرف

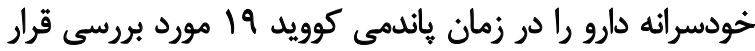

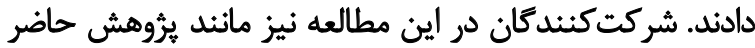

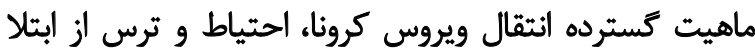

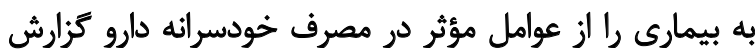

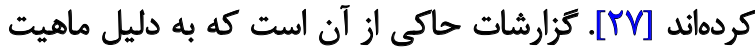

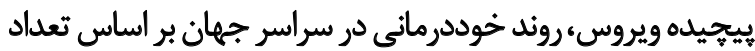

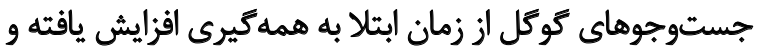

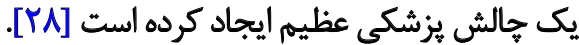

يكى ديكر ازعوامل مؤثر در خوددرمانى، قرنطينه خانكى بود بوني

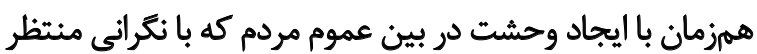

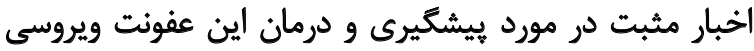

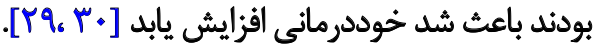
مطالعه ماكوسكاو همكاران همجنين نشان داد از زمان شروع دارئ

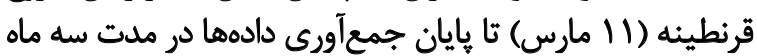

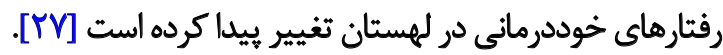
در مطالعه اوكان و همكاران، داشتن اطلاعات دارويى، تجربه

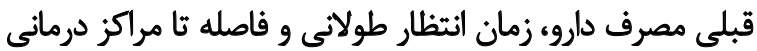

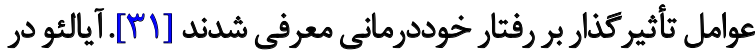

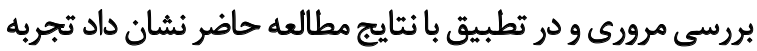

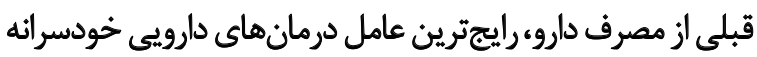

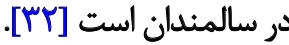

آنجهه در ميان يُروهشهاى مختلف مشترك است، تجربه قبلى

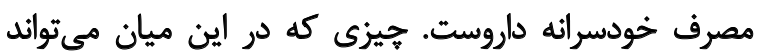

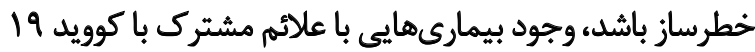

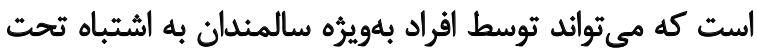

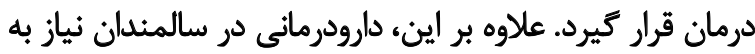

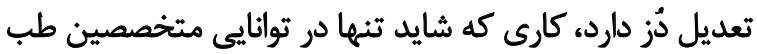

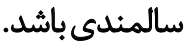

تعداد روزافزون مبتلايان به ويروس كروناو افزايش مراجعات به

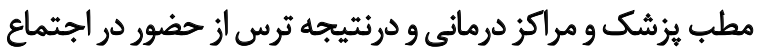

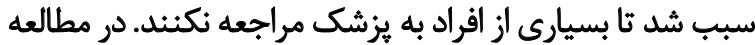

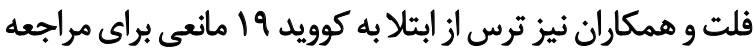

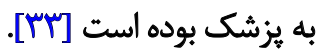

يافتههاى مطالعه نيكولز و همكاران نشان داد در افراد باد

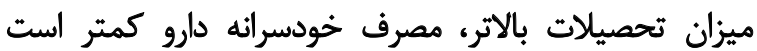

اطلاعات دارويى كافى در مورد نوع داروى مصرفى داشتند و آكاهى

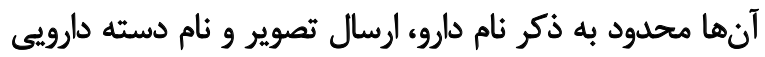

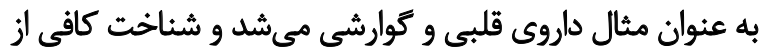

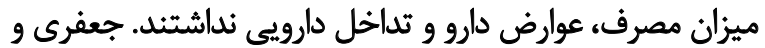

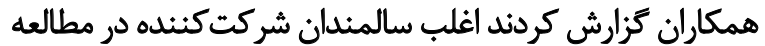

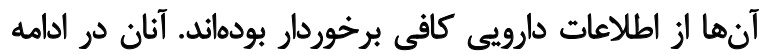

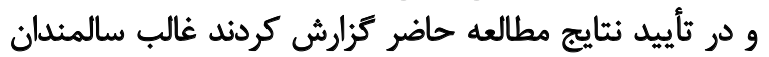
اين مطالعه از داروهاى موجود در منازل استفاده مي كنيند [1/11]

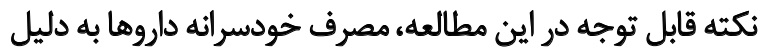

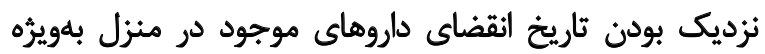

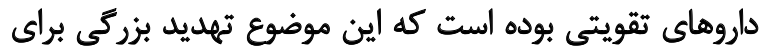

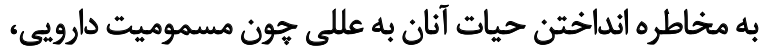

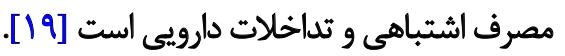
از رايجترين داروهاى مصرفى در درمانهاى خودسرانه

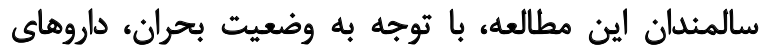

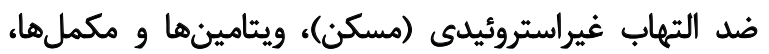

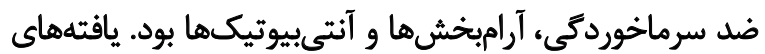

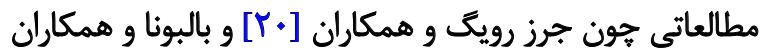

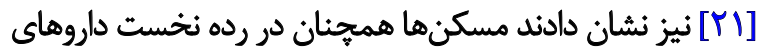

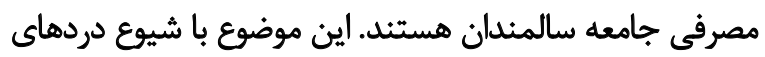

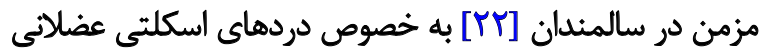

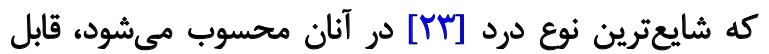

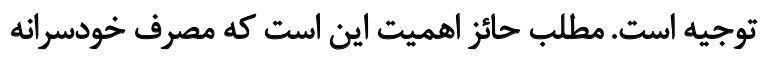

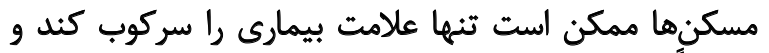

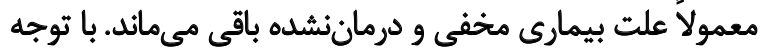

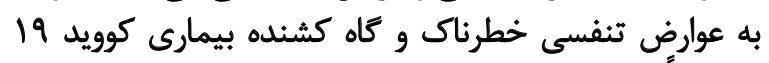

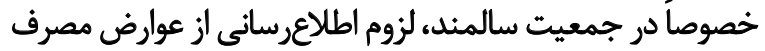
خودسرانه دارو بهويره مسكن ها ضرورى به نظر ميرسد.

از سويى ديكر، مطالعات صورت كرفته در زمينه كوويد 19

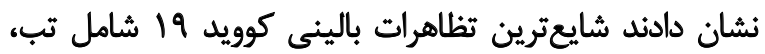

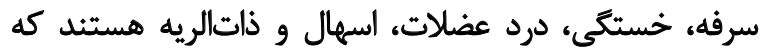

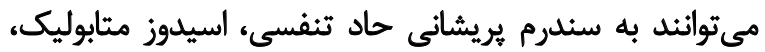

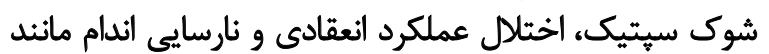

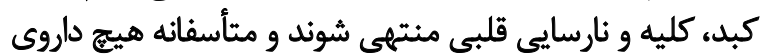

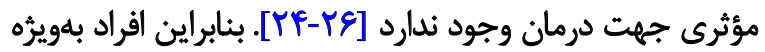

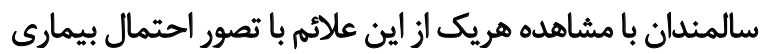

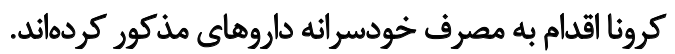
يراكندكى و حجم اطلاعات غلط، ماندكارى بيشتر در منزل

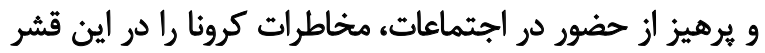

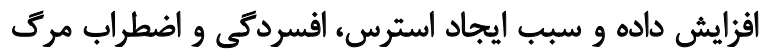

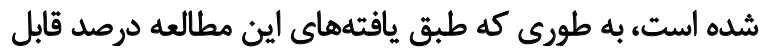

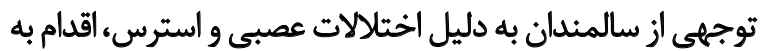
مصرف خودسرانه آرامبخشهان بهر الير اخداندات 


\section{نتيجهيَيرىنهايى}

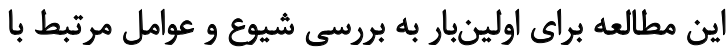

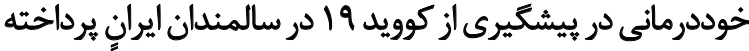

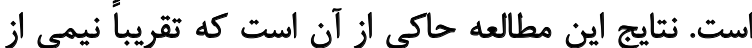

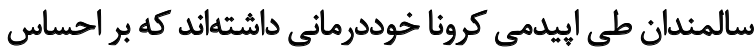

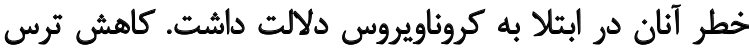

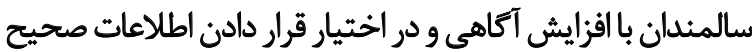

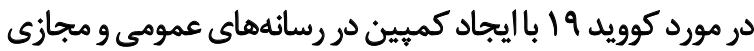

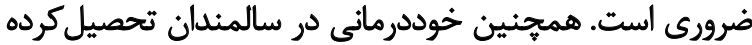

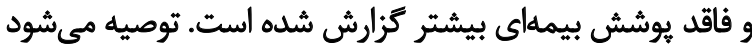

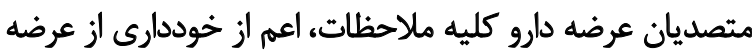

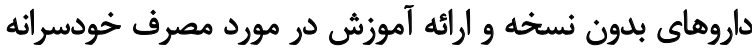

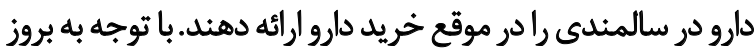

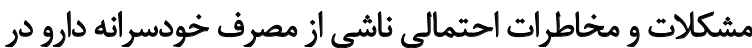

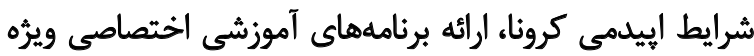

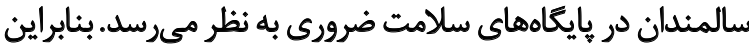

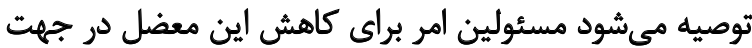
ارتقاى سطح آكاهى سالمندان اقدام كنئد.

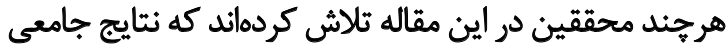

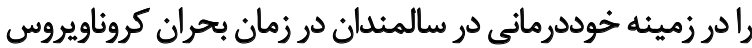

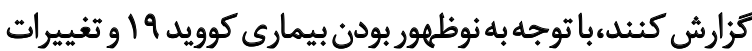

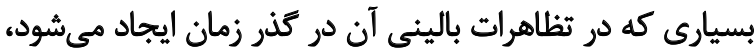

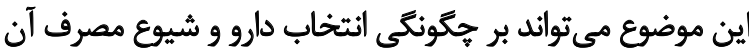

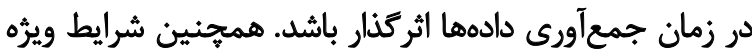

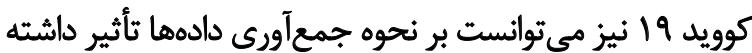

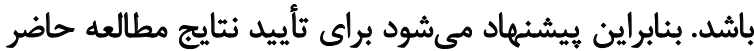

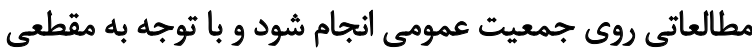

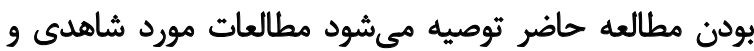
كوهورت جهت بررسى التوى مصرف دارو و ميزان ابتلا به كوويد 19 و وشدث علائم آن در سالمندان انجام شود.

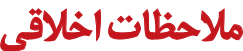

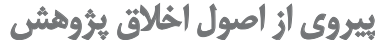

اين يروهش در كميته اخلاق دانشاه علوميزشكى سبزوار بررسى

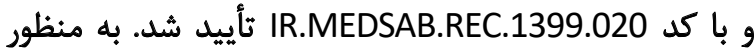

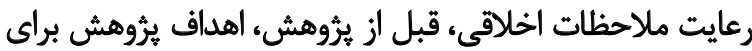

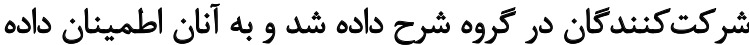

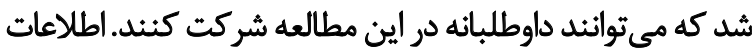

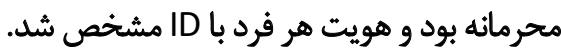

[IV] [ مطالعاى در لبنان نيز نشان داد خوددرمانى به طور قابل

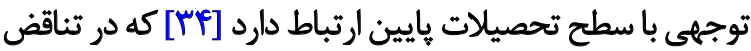

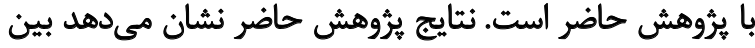

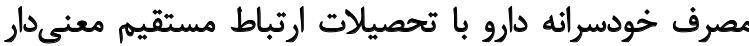

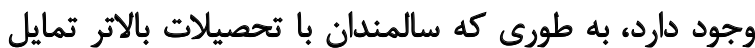

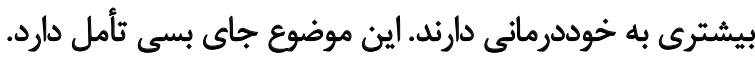

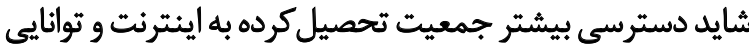

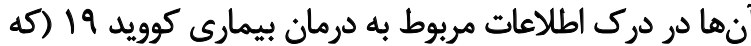

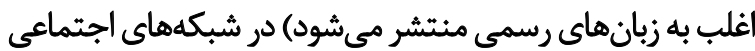
و تجويز و فروش محصولات دارويى مقابله با بيمارى بتواند اين اين روند راتوضيح دهد.

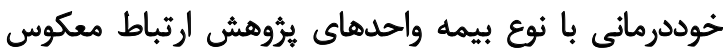

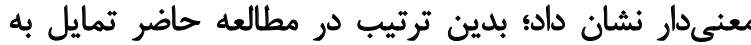

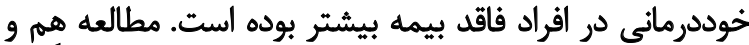

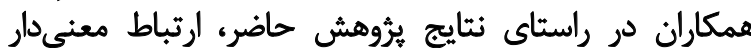

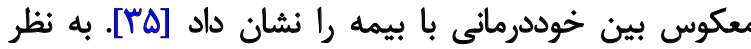

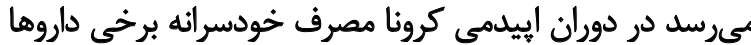

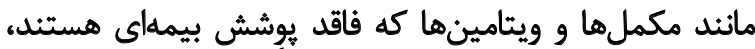

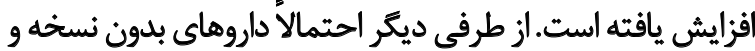

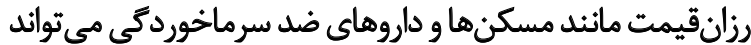
يكى ديكر از علل خوددرمانى در سالمندان باشد.

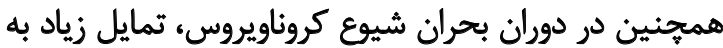

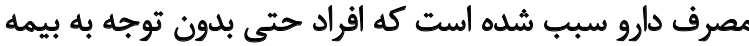
خود اقدام به مصرف خودسرانه دارو كنيد.

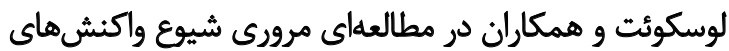

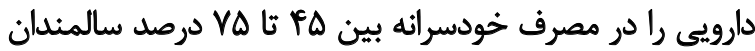

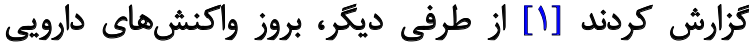

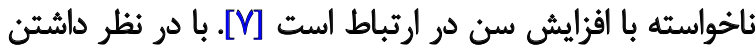

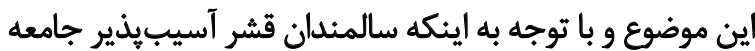
محسوب مىشوضند، برواضح است كه در مر مقايسه با با ساير اقشار جامعه در معرض خطرات بيشترى در مواجهه با خوددرمانى

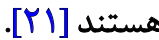

كشور ايران در آيندالى نزديك با جامعه بزركى از سالمند

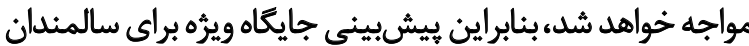

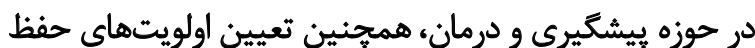

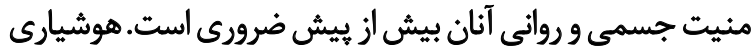

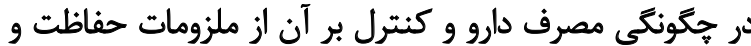

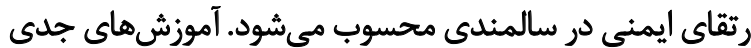

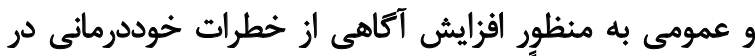

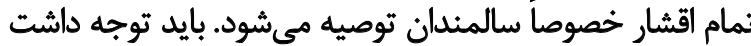

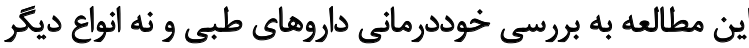

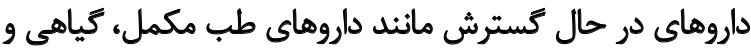
سنتى يرداخته است. 


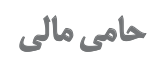

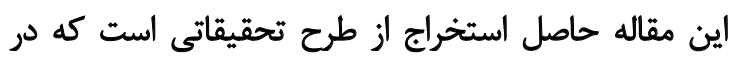

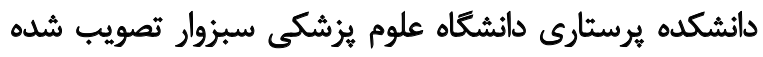

$$
\text { است. }
$$

$$
\text { مشاركت ثويسندكان }
$$

ايدهردازى و نظارت، نكارش، بررسى و ويرايش: نرجس

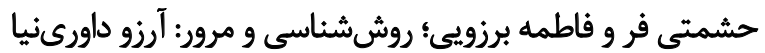

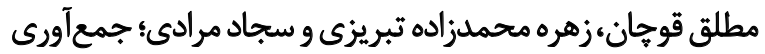

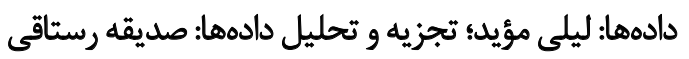

$$
\text { ت تعارض منافع }
$$

بنابر اظهار نويسندكان اين مقاله هيجكونه تعارض منافعى

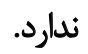

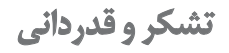

از تمامى سالمندانى كه در يُروهش ما شركت كردند، همجنين مديران و مسئولين و اساتيد در دانشكده يرستاني سبزوار و مراكز بهداشتى شهر سبزوار تشكر و قدردانى مى شئود. 


\section{Reference}

[1] Locquet M, Honvo G, Rabenda V, Van Hees T, Petermans J, Reginster JY, et al. Adverse health events related to self-medication practices among elderly: A systematic review. Drugs \& Aging. 2017; 34(5):359-65. [DOI:10.1007/s40266-017-0445-y] [PMID]

[2] Montastruc JL, Bondon-Guitton E, Abadie D, Lacroix I, Berreni A, Pugnet G, et al. Pharmacovigilance, risks and adverse effects of self-medication. Therapies. 2016; 71(2):257-62. [DOI:10.1016/j. therap.2016.02.012] [PMID]

[3] Korani TAT, Darvishporkakhki A, Shahsavari S, Esmaeli R. [Assessment of selfmedication and associated factors among elderly living in Kermanshah city in 2014 (Persian)]. Journal of Geriatric Nursing. 2015; 3(1):38-48. [DOI:10.21859/jgn.3.1.38]

[4] Chouhan K, Prasad SB. Self-medication and their consequences: A challenge to health professional. Asian Journal of Pharmaceutical and Clinical Research. 2016; 9(2):314-7. https:// www.researchgate.net/ profile/Dr-Shyam-Prasad/publication/302959170_Self-medication_and_their_ consequences_A_challenge_to_health_professional/ links/5734493908ae298602dcff89/Self-medication-and-their-consequences-A-challenge-to-health-professional.pdf

[5] Ghorbani M, Ghanei Gheshlagh R, Dalvand S, Moradi B, Faramarzi P, Moradi MZ. [Evaluation of self-medication and related factors in elderly population of Sanandaj, Iran (Persian)]. Scientific Journal of Nursing, Midwifery and Paramedical Faculty. 2019; 4(4):46-57. http://sjnmp.muk.ac.ir/article-1-188-en.html

[6] Zhang X, Zhou S, Zhou Y, Liu X. [Research progress in inappropriate drug use in the elderly (Chinese)]. Chinese Journal of Geriatrics. 2018; 37(4):479-84. http://wprim.whocc.org.cn/admin/ article/articleDetail?WPRIMID=709288\&articleId=709773

[7] Maher RL, Hanlon J, Hajjar ER. Clinical consequences of polypharmacy in elderly. Expert Opinion on Drug Safety. 2014; 13(1):57-65. [DOI:10.1517/14740338.2013.827660] [PMID] [PMCID]

[8] Shenoy P, Harugeri A. Elderly patients' participation in clinical trials. Perspectives in Clinical Research. 2015; 6(4):184-9. [DOI:10.4103/2229-3485.167099] [PMID] [PMCID]

[9] Novel CP. [The epidemiological characteristics of an outbreak of 2019 novel coronavirus diseases (COVID-19) in China (Chinese)]. Zhonghua liu xing bing xue za zhi= Zhonghua liuxingbingxue zazhi. 2020; 41(2):145-51. [DOI:10.46234/ccdcw2020.032]

[10] Recalcati S. Cutaneous manifestations in COVID_19: A first perspective. Journal of the European Academy of Dermatology and Venereology. 2020; 34(5):e212-3. [DOI:10.1111/jdv.16387]

[11] Tan L, Wang Q, Zhang D, Ding J, Huang Q, Tang YQ, et al. Lymphopenia predicts disease severity of COVID-19: A descriptive and predictive study. Signal Transduction and Targeted Therapy. 2020; 5:33. [DOI:10.1038/s41392-020-0159-1] [PMCID]

[12] Liu K, Chen Y, Lin R, Han K. Clinical features of COVID-19 in elderly patients: A comparison with young and middle-aged patients. Journal of Infection. 2020; 80(6):e14-8. [DOI:10.1016/j. jinf.2020.03.005] [PMID] [PMCID]

[13] Airagnes G, Pelissolo A, Lavallée M, Flament M, Limosin F. Benzodiazepine misuse in the elderly: Risk factors, consequences, and management. Current Psychiatry Reports. 2016; 18(10):89. [DOI:10.1007/s11920-016-0727-9] [PMID]
[14] Sundel M, Sundel SS. Behavior change in the human services: Behavioral and cognitive principles and applications. California: Sage Publications; 2017. https://us.sagepub.com/en-us/nam/ behavior-change-in-the-human-services/book245199

[15] Ribeiro DC, Milosavljevic S, Abbott JH. Sample size estimation for cluster randomized controlled trials. Musculoskeletal Science and Practice. 2018; 34:108-11. [DOI:10.1016/j.msksp.2017.10.002] [PMID]

[16] Masumoto S, Sato M, Maeno T, Ichinohe Y, Maeno T. Factors associated with the use of dietary supplements and over-thecounter medications in Japanese elderly patients. BMC Family Practice. 2018; 19:20. [DOI:10.1186/s12875-017-0699-9] [PMID] [PMCID]

[17] Niclós G, Olivar T, Rodilla V. Factors associated with self_medication in Spain: A cross_sectional study in different age groups. International Journal of Pharmacy Practice. 2018; 26(3):258-66. [DOI:10.1111/ijpp.12387] [PMID]

[18] Jafari F, Khatony A, Rahmani E. Prevalence of self-medication among the elderly in Kermanshah-Iran. Global Journal of Health Science. 2015; 7(2):360-5. [DOI:10.5539/gjhs.v7n2p360]

[19] Gracia-Vásquez SL, Ramírez-Lara E, Camacho-Mora IA, Cantú-Cárdenas LG, Gracia-Vásquez YA, Esquivel-Ferriño PC, et al. An analysis of unused and expired medications in Mexican households. International Journal of Clinical Pharmacy. 2015 37(1):121-6. [DOI:10.1007/s11096-014-0048-1] [PMID]

[20] Jerez-Roig J, Medeiros LF, Silva VA, Bezerra CL, Cavalcante LA, Piuvezam G, et al. Prevalence of self-medication and associated factors in an elderly population: A systematic review. Drugs Aging. 2014; 31(12):883-96. [DOI:10.1007/s40266-014-0217-x] [PMID]

[21] Balbuena FR, Aranda AB, Figueras A. Self-medication in older urban mexicans. Drugs \& Aging. 2009; 26(1):51-60 [DOI:10.2165/0002512-200926010-00004] [PMID]

[22] Murphy N, Karlin-Zysman C, Anandan S. Management of chronic pain in the elderly: A review of current and upcoming novel therapeutics. American Journal of Therapeutics. 2018; 25(1): e36-43. [DOI:10.1097/MJT.0000000000000659]

[23] Rapo-Pylkkö S, Haanpää M, Liira H. Chronic pain among community-dwelling elderly: A population-based clinical study. Scandinavian Journal of Primary Health Care. 2016; 34(2):159-64. [DOI:10.3109/02813432.2016.1160628] [PMID] [PMCID]

[24] Wang D, Hu B, Hu C, Zhu F, Liu X, Zhang J, et al. Clinical characteristics of 138 hospitalized patients with 2019 novel coronavirus-infected pneumonia in Wuhan, China. JAMA. 2020; 323(11):1061-9. [DOI:10.1001/jama.2020.1585] [PMID] [PMCID]

[25] Huang C, Wang Y, Li X, Ren L, Zhao J, Hu Y, et al. Clinical features of patients infected with 2019 novel coronavirus in Wuhan, China. Lancet. 2020; 395(10223):497-506. [DOI:10.1016/S01406736(20)30183-5]

[26] Chen N, Zhou M, Dong X, Qu J, Gong F, Han Y, et al. Epidemiological and clinical characteristics of 99 cases of 2019 novel coronavirus pneumonia in Wuhan, China: A descriptive study. Lancet. 2020; 395(10223):507-13. [DOI:10.1016/S0140-6736(20)30211-7]

[27] Makowska M, Boguszewki R, Nowakowski M, Podkowińska M. Self-medication-related behaviors and Poland's COVID-19 lockdown. International Journal of Environmental Research and Public Health. 2020; 17(22):8344. [DOI:10.3390/ijerph17228344] [PMID] [PMCID] 
[28] Onchonga D. A Google Trends study on the interest in selfmedication during the 2019 novel coronavirus (COVID-19) disease pandemic. Saudi Pharmaceutical Journal. 2020; 28(7):903-4. [DOI:10.1016/j.jsps.2020.06.007] [PMID] [PMCID]

[29] Sadio AJ, Gbeasor-Komlanvi FA, Konu RY, Bakoubayi AW, Tchankoni MK, Bitty-Anderson AM, et al. Assessment of selfmedication practices in the context of the COVID-19 outbreak in Togo. BMC Public Health. 2021; 21:58. [DOI:10.1186/s12889-02010145-1] [PMID] [PMCID]

[30] Quispe-Cañari JF, Fidel-Rosales E, Manrique D, Mascaró-Zan J, Huamán-Castillón KM, Chamorro-Espinoza SE, et al. Self-medication practices during the COVID-19 pandemic among the adult population in Peru: A cross-sectional survey. Saudi Pharmaceutical Journal. 2020 ;29(1):1-11. [DOI:10.2139/ssrn.3688689]

[31] Ocan M, Bwanga F, Bbosa GS, Bagenda D, Waako P, OgwalOkeng J, et al. Pattern and predictor of self medication in northern Uganda. Plos One. 2014; 9(3):e92323 [DOI:10.1371/journal. pone.0092323] [PMID] [PMCID]

[32] Ayalew MB. Self-medication practice in Ethiopia: A systematic review. Patient Preference and Adherence. 2017; 11:401-13 [DOI:10.2147/PPA.S131496] [PMID] [PMCID]

[33] Flett GL, Heisel MJ. Aging and feeling valued versus expendable during the COVID-19 pandemic and beyond: A review and commentary of why mattering is fundamental to the health and well-being of older adults. International Journal of Mental Health and Addiction. 2020:1-27. [DOI:10.1007/s11469-020-00339-4]

[34] Jamhour A, El-Kheir A, Salameh P, Hanna PA, Mansour H. Antibiotic knowledge and self-medication practices in a developing country: A cross-sectional study. American Journal of Infection Control. 2017; 45(4):384-8. [DOI:10.1016/j.ajic.2016.11.026] [PMID]

[35] Hem E, stokke G, Cronvold NT. Self- prescribing among young Norwegian doctors: A nine year followup study of a nation with sample. BMC Medicine. 2005; 3:16. [DOI:10.1186/1741-7015-3-16] [PMID] [PMCID] 